\title{
Femoral neck fracture following intramedullary nailing with misplacement of an end cup: report of two cases
}

\author{
Paweł Grala $\cdot$ Bartosz Mańkowski · \\ Grzegorz Kierzynka
}

Received: 29 February 2008/Accepted: 8 November 2008/Published online: 12 December 2008

(C) Springer-Verlag 2008

\begin{abstract}
Femoral neck fracture is an unusual complication of intramedullary fixation of a broken femur. We report on two cases of femoral neck fractures attributed to misplacement of an end cup and subsequent invasive maneuvers in an effort to remove it. Iatrogenic fractures of the femoral neck during or after intramedullary nailing are reported in the medical literature. Authors associate it with many possible technical mistakes performed during the procedure, yet no complications after missed end cup placement were noted. We suggest that the fractures described below were a consequence of injury to the vascular supply and bone stock of the initially intact femoral neck.
\end{abstract}

Keywords Femoral neck fracture .

Intramedullary nailing

\section{Introduction}

Over the last three decades closed intramedullary nailing has become the gold standard technique for the treatment of femoral shaft fractures. Its value has been proven by a high union rate, low number of complications, and low invasiveness to the soft tissues around the femur. The procedure is still technically demanding, so modern intramedullary nails with their insertion instruments are increasingly surgeon and patient friendly. Between 1995 and 2007, over 400 patients with femoral fractures were treated in our department with various types of antegrade

P. Grala $(\bowtie) \cdot$ B. Mańkowski · G. Kierzynka

Department of Trauma, Burns and Plastic Surgery,

Karol Marcinkowski Medical University, Poznan, Poland

e-mail: pawel.grala@aoalumni.org cannulated locked femoral nails, but until recently we did not recognize any femoral neck fracture (FNF) associated with this procedure. We position patients on a fracture table using foot straps to provide longitudinal traction. Countertraction is applied via a perineal post. We always use the piriform fossa for the nail entry site. Since 2005 we have followed two cases with unusual complications attributed, in our opinion, to technical errors during the end stage of the procedure - end cup placement. The two senior authors fully recollect the procedure due to technical intraoperative complications. In both cases Synthes universal femoral nails were used. Immediately postoperative $\mathrm{X}$-rays did not reveal signs of FNF, but on first follow-up after two weeks both patients were symptomatic, and anteroposterior (ap) radiograms revealed misplaced end cups and FNF.

\section{Case reports}

Both patients presented below were informed about the nature of this report and gave their consent to its publication.

\section{Case 1}

A 76-year-old obese woman (BMI 32) was referred to our department after infected destabilization of a plate fixation of the left femoral shaft. On the next day she underwent removal of the implant with debridement and fixation of the bone with ZESPOL internal fixator-a Polish harbinger for the locked plates (Fig. 1). On the third postoperative day she sustained another fracture of the left femur while the wound dressings were changed. During an operation carried out on the next day, the fixator was lengthened 


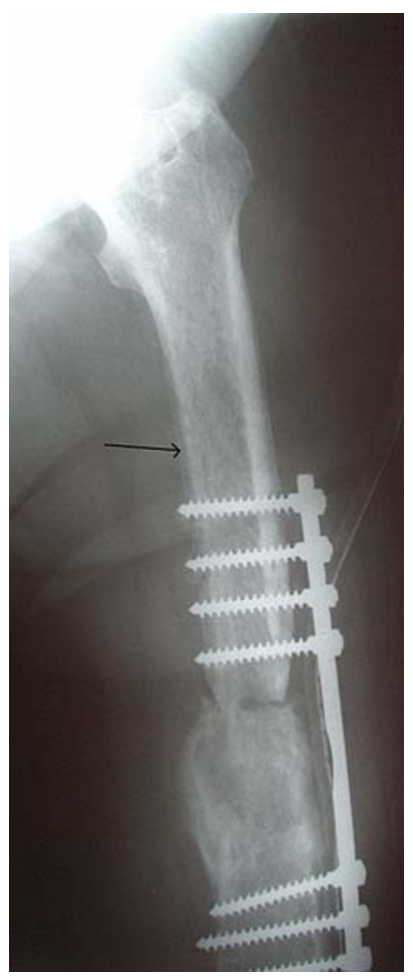

Fig. 1 Case 1: ZESPOL internal fixator after removal of an infected plate. Proximal fragment periosteal elevation (black arrow) suggesting an inflammatory reaction

proximally to incorporate the proximal fragment (Fig. 2). After three months, despite non-weight-bearing, recurrent destabilization occurred without any microbiological symptoms of infection. The implant was removed and open intramedullary nailing was performed. During this procedure significant bone fragility was evident, and the end cup was misplaced behind the femoral neck. Multiple efforts to remove it (using a finger and vascular clamps under the image intensifier) failed. After two weeks, during the first follow-up visit, a FNF (AO 31-B2) was diagnosed but the patient refused the proposed operative treatment. She came back after three months with significant shortening of the left femur and nonunions of both the neck and diaphysis (Fig. 3). During another operative intervention the end cup was replaced, and fixation of the femoral neck was attempted with two screws, one cortical interlocking through the dynamic slot of the nail and another cancellous with the "miss a nail technique." We were not able to place additional screws due to the width of the proximal nail. The soft bone of the proximal fragment did not cause any noticeable resistance during screw placement. After three days of bed rest, the screws redisplaced and were removed. The patient remains severely disabled, walking around the house with a walker and occasionally using a Thomas splint based on her pelvis.

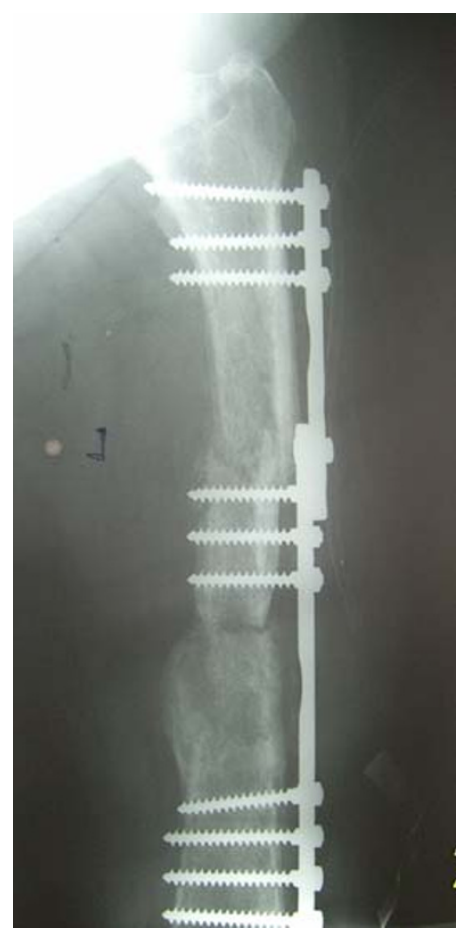

Fig. 2 Case 1: extended ZESPOL fixator after additional fracture was sustained during in-bed patient care. Apparently healthy femoral neck

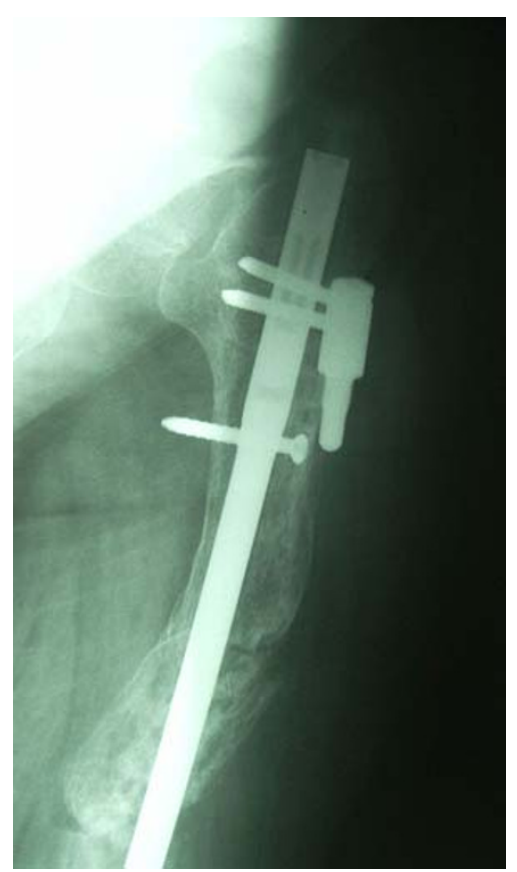

Fig. 3 Case 1: nonunion of the femoral neck and femoral diaphysis

Case 2

A 68-year-old obese woman (BMI 30) was admitted after a fall at home causing fracture of the femoral shaft (Figs. 4,5). On the same day she had reamed intramedullary fixation of 


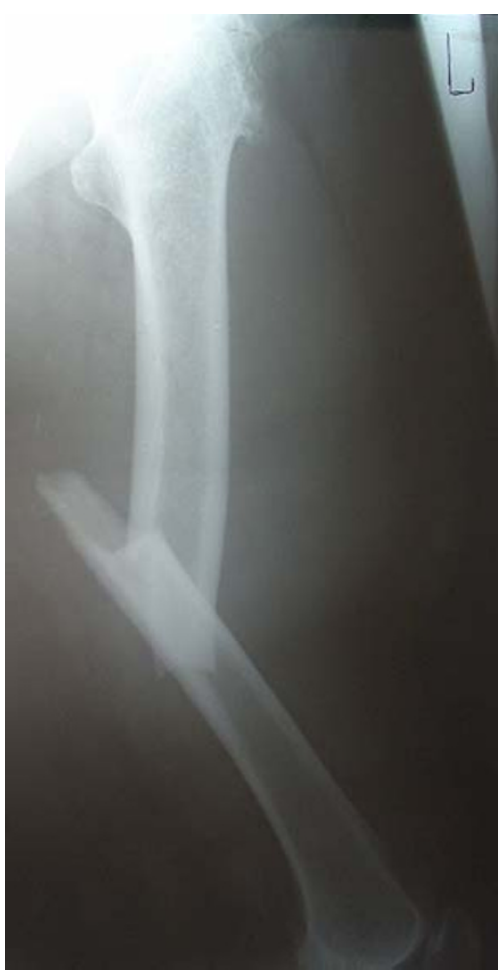

Fig. 4 Case 2: broken femoral shaft

the femur performed. The nail was short and thus advanced deeper. Except at the end stage, no technical difficulties were noticed. The end cup was placed inside the femoral neck and efforts to remove it with a vascular clamp failed. Immediate postoperative radiogram did not reveal FNF (Fig. 6).

The patient presented with symptoms two weeks after discharge. X-ray examination revealed an AO 31-B3 FNF (Fig. 7). The patient was reoperated after seven weeks with symptoms of bone union of the femoral shaft. The nail was removed and bipolar arthroplasty of the hip was performed (Fig. 8). The subsequent postoperative course was uneventful.

\section{Discussion}

We assume that both of the above-described FNFs were iatrogenic and related to harmful maneuvers during efforts to remove the misplaced end cup. The damage to circumflex vessels in case 1 and to endosteal blood supply and bone stock itself in case 2 resulted in late FNF. Apart from this suggestion, in case 1 the weakened, ischemic bone failed during physiologic stresses at bed rest after first fixation with ZESPOL, so we also consider the possibility of a similar cause for FNF.

Cases of FNF after intramedullary nailing have also been described by other authors. The proposed causes vary from misplacement of the entry point to forceful removal

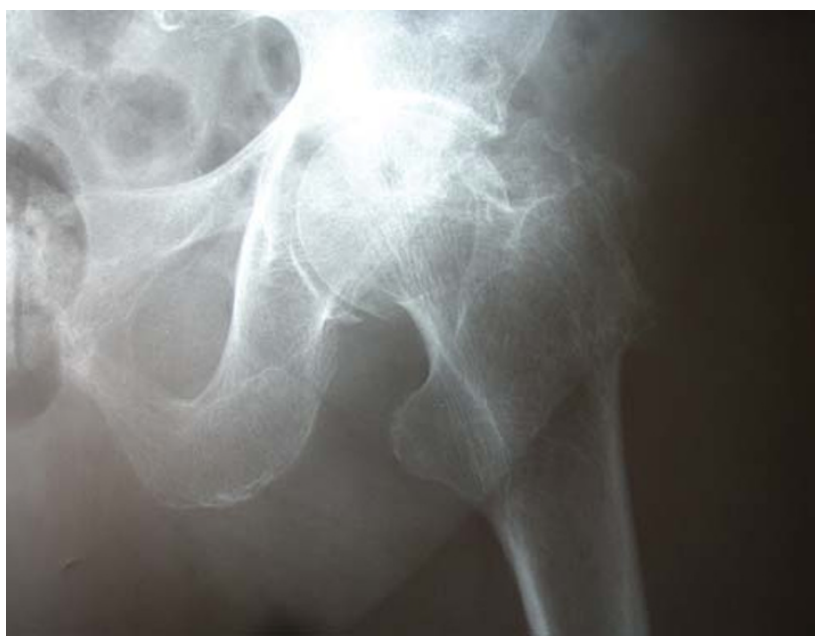

Fig. 5 Magnified view of the femoral neck region (same radiogram as in Fig. 4)-apparently healthy femoral neck

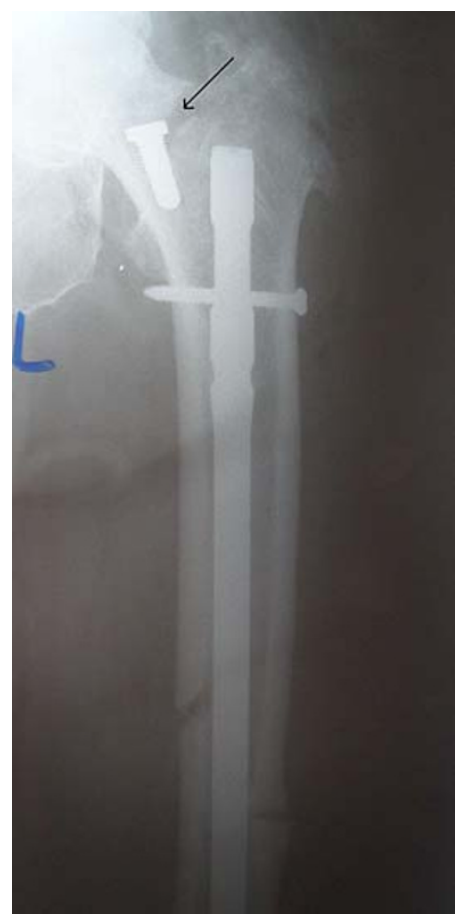

Fig. 6 Case 2: misplacement of an end cup. Visible iatrogenic injury to the base of the femoral neck (black arrow). Medial cortex of the femoral neck apparently intact

of a drill jammed into the femoral shaft [1]. Various types of fractures are reported, but the classification is inconsistent (Pauwels III, comminuted transverse, comminuted segmental, etc.).

Khan [2] found three patients with postoperatively identified FNF. All were treated conservatively (due to minimal displacement) and resulted in various malunions in two cases. He attributed the fracture to forceful insertion of the initiating awl in the wrong direction and the use of 


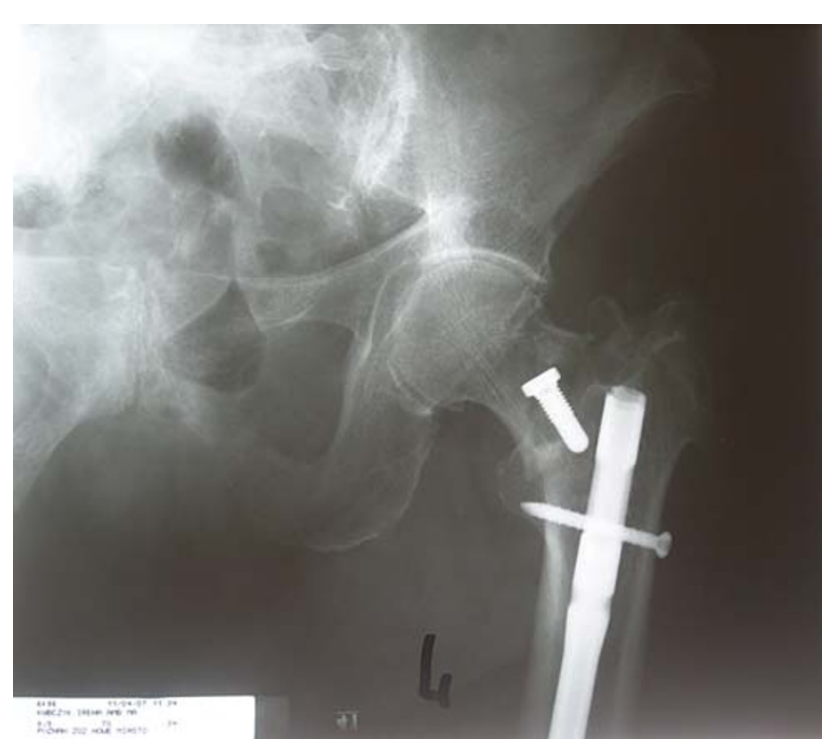

Fig. 7 Case 2: iatrogenic femoral neck fracture

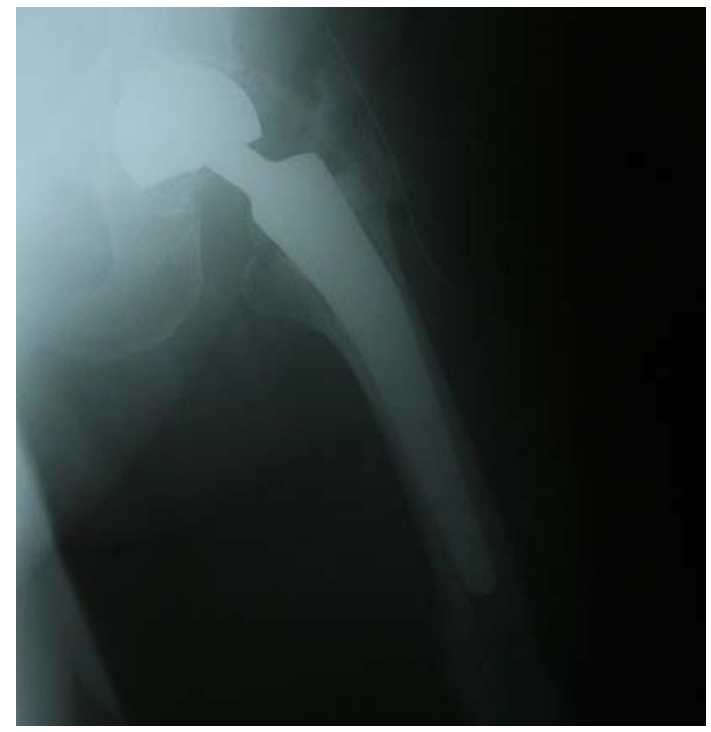

Fig. 8 Case 2: bipolar arthroplasty of the hip after intramedullary nail removal

multiple entry points (like Harper) [3]. Christie and CourtBrown [4] reported four (of 143 treated) extracapsular FNFs during closed femoral intramedullary nailing, which were attributed to an excessively lateral insertion point in the trochanteric area and to the oblique insertion of the nail. In a laboratory study of strain gauge locations on cadaver bones, researchers from the AO Research Institute found that antegrade femoral nails, introduced both into the piriformis entry point or laterally, do not induce higher strains if correspondingly designed nails are implanted the correct way. Deviations from these ideal insertion points are associated with higher stains and sometimes fractures in the proximal femur [5].

Accordingly, in obese patients, difficult access to the piriformis entry point might cause additional stress to the femoral neck if the ideal site for nail insertion is missed. Such a cause must also be taken into account in both cases described above.

Simonian [6] had four cases of iatrogenic FNF which he attributed to overhanging of the medial prong of the old type of AO insertion jig that may impinge on the superior aspect of a valgus femoral neck during the final stage of a deep nail impaction. Another unusual case was due to nail insertion with a reversed curvature (posterior bowing) [7].

We currently use Synthes reamed femoral nails, where the introductory device fits the nail's diameter perfectly, eliminating the circumferential overhanging that was blamed for the fracture in Simonian's cases [7].

End cup misplacement errors are common, especially in obese individuals if the piriformis entry site is used, but consequences such as those described in this manuscript have not been reported previously in the literature. We currently tie the end cup with an absorbable suture prior to its placement, so that it is easy to recover the device in the event of misplacement without the need for any additional use of fluoroscopy, loss of time and injuries to adjacent structures, which could end up with FNF.

Conflict of interest statement We declare that we have no conflict of interest related to the publication of this manuscript.

\section{References}

1. Deep K, Sharp I, Hav SM (1999) Femoral neck fracture complicating intramedullary nailing of femoral shaft. Injury 30:445-447

2. Khan FA, Ikram MA, Badr AA, Al-Khawashki H (1995) Femoral neck fracture: a complication of femoral nailing. Injury 26(5):319321

3. Harper MC, Henstorf J (1986) Fractures of the femoral neck associated with technical errors in closed intramedullary nailing of the femur. Report of two cases. J Bone Joint Surg Am 68-A:624626

4. Christie J, Court-Brown C (1988) Femoral neck fracture during closed medullary nailing: brief report. J Bone Joint Surg Br 70B:670-673

5. Schwieger K, Moein CA, Bösl O, Verhofstad M (2007) Lateral insertion points in antegrade femoral nailing and their influence on femoral bone strains. AO Dev News 1:48-49

6. Simonian PT, Chapman JR, Seleznick HS, Bernischke SK, Claudi BF, Swiontkowski MF (1994) Iatrogenic fractures of the femoral neck during closed nailing of the femoral shaft. J Bone Joint Surg Br 76-B:293-296

7. Apivatthakakul T, Arpornchayanon O (2001) Iatrogenic femoral neck fracture caused by mal-insertion of a curved intramedullary nail. Injury 32:727-729 